\title{
Evaluation of peak inspiratory pressure, tidal volume and respiratory rate during ventilation of premature lambs using a self-inflating bag
}

\author{
Avaliação do pico de pressão, do volume corrente e da freqüência respiratória \\ durante ventilação de carneiros prematuros utilizando balão auto-inflável \\ Jefferson G. Resende ${ }^{1}$, Carlos A. M. Zaconeta ${ }^{2}$, Antonio C. P. Ferreira ${ }^{3}$, \\ César A. M. Silva ${ }^{4}$, Marcelo P. Rodrigues ${ }^{5}$, Celso M. Rebello6, Paulo Tavares ${ }^{7}$
}

\section{Resumo}

Objetivo: Avaliar o pico de pressão inspiratória, o volume corrente e a freqüência respiratória obtidos durante ventilação manual de carneiros prematuros, utilizando balão auto-inflável.

Métodos: Estudo experimental descritivo em que cinco duplas de médicos selecionados aleatoriamente entre 35 neonatologistas que trabalham em unidade de terapia intensiva neonatal e experientes em reanimação de recém-nascidos ventilaram cinco carneiros prematuros intubados, utilizando balão auto-inflável. Os sinais de pressão e fluxo eram captados, convertidos por meio de transdutores e digitalizados para armazenamento e análise. Foram avaliadas curvas de pressão e de volume corrente, este a partir da integral do fluxo, em suas medidas de pico, nos 50 segundos finais de cada 5 minutos.

Resultados: A mediana da pressão foi de 39,8 ( $\mathrm{IQ}_{25-75 \%} 30,2-$ $47,2) \mathrm{cmH}_{2} \mathrm{O}$; foi menor que $20 \mathrm{em} 1,1 \%$ das vezes e maior que $40 \mathrm{em}$ $49,1 \%$. Sete em 10 médicos propiciaram mais de seis picos de pressão maiores que $40 \mathrm{cmH}_{2} \mathrm{O}$. A mediana do volume corrente/ $\mathrm{kg}$ foi de 17,8 $\left(\mathrm{IQ}_{25-75 \%} 14,1-22,4\right) \mathrm{mL}$, sendo menor que $5 \mathrm{~mL}$ em $0,1 \%$ das vezes e igual ou maior que $20 \mathrm{~mL}$ em $37,7 \%$. Todos os médicos impuseram cinco ou mais ciclos ventilatórios com volume corrente $/ \mathrm{kg}$ de $20 \mathrm{~mL}$ ou mais. A freqüência situou-se entre 30 e 60 ciclos/minuto em $65,9 \%$ das vezes, sendo menor que $30 \mathrm{em} 6,8 \%$ e maior que $60 \mathrm{em} 27,3 \%$ das vezes.

Conclusão: Ocorreu grande variabilidade nos valores do pico de pressão inspiratória e do volume corrente $/ \mathrm{kg}$, sendo muitas vezes elevados e alcançando níveis indutores de biotrauma; para a freqüência respiratória, os valores foram adequados na maioria das vezes.

J Pediatr (Rio J). 2006;82(4):279-83: Ressuscitação cardiopulmonar, ventilação mecânica, recém-nascido, asfixia neonatal, volume de ventilação pulmonar.

\section{Abstract}

Objective: To evaluate the peak inspiratory pressure, tidal volume and respiratory rate achieved during manual ventilation of premature lambs, using a self-inflating bag.

Methods: In this descriptive, experimental study, five pairs of physicians, selected at random among 35 neonatologists working at a neonatal intensive care unit and with experience in the resuscitation of newborn infants, ventilated five intubated premature lambs using a self-inflating bag. Pressure and flow monitor signals were passed through a transducer and digitized for recording and analysis. Tidal volume and pressure curves were obtained from the integral of flow rate, at peak, during the last 50 seconds of every fifth minute, and analyzed.

Results: Median pressure was $39.8\left(\mathrm{IQ}_{25-75 \%} 30.2-47.2\right) \mathrm{cmH}_{2} \mathrm{O}$; being below 20 in $1.1 \%$ of cases and above 40 in $49.1 \%$. Seven out of 10 physicians produced more than six pressure peaks of over 40 $\mathrm{cmH}_{2} \mathrm{O}$. Median tidal volume/kg was 17.8 ( $\left.\mathrm{IQ}_{25-75 \%} 14.1-22.4\right) \mathrm{mL}$, being below $5 \mathrm{~mL}$ in $0.1 \%$ of cases and greater than or equal to $20 \mathrm{~mL}$ in $37.7 \%$. All of the physicians propelled five or more ventilation cycles with tidal volume $/ \mathrm{kg}$ of $20 \mathrm{~mL}$ or more. Respiratory rate was between 30 and $60 \mathrm{cycles} / \mathrm{minute}$ in $65.9 \%$ of cases, being below 30 in $6.8 \%$ of cases and over 60 in $27.3 \%$ of cases.

Conclusions: There was major variation in peak inspiratory pressure and tidal volume/kg values, which were in many cases elevated, attaining levels that habitually cause biotrauma, while respiratory rates were adequate in the majority of cases.

J Pediatr (Rio J). 2006;82(4):279-83: Cardiopulmonary resuscitation, mechanical ventilation, neonate, neonatal asphyxia, tidal volume.

1. Médico pediatra. Doutorando em Ciências Médicas, Universidade de Brasília (UnB), Brasília, DF.

2. Médico neonatologista. Mestre, UnB, Brasília, DF.

3. Médico intensivista pediátrico. Mestre, Universidade Federal de São Paulo (UNIFESP), São Paulo, SP.

4. Fisioterapeuta. UnB, Brasília, DF. Fisioterapeuta, Hospital Universitário da UnB, Brasília, DF.

5. Médico pneumologista. Mestre, UnB, Brasília, DF. Professor, UnB, Brasília, DF.

6. Médico. Doutor, Faculdade de Medicina, Universidade de São Paulo (USP), São Paulo, SP. Coordenador, Unidade de Pesquisa Experimental, Instituto da Criança do Hospital das Clínicas, Faculdade de Medicina, USP, São Paulo, SP.

7. Médico pneumologista. Doutor. Professor emérito, UnB, Brasília, DF.

Apoio financeiro: O estudo foi realizado no Laboratório de Fisiologia do Aparelho Respiratório da Universidade de Brasília (UnB), em Brasília, DF, com a colaboração do Departamento de Obstetrícia de Ovinos da Faculdade de Medicina Veterinária da UnB. Os berços aquecidos foram cedidos por Olidef CZ, além de recursos próprios dos autores. O presente estudo é parte da tese de doutorado do autor Jefferson Guimarães de Resende, intitulada "Avaliação da mecânica ventilatória, dos gases arteriais e das lesões pulmonares durante a ventilação pulmonar mecânica manual de carneiros prematuros, comparando dois ventiladores manuais".

Artigo submetido em 25.08.05, aceito em 10.05.06.

Como citar este artigo: Resende JG, Zaconeta CA, Ferreira AC, Silva CA, Rodrigues MP, Rebello CM, et al. Evaluation of peak inspiratory pressure, tidal volume and respiratory rate during ventilation of premature lambs using a self-inflating bag. J Pediatr (Rio J). 2006;82:279-83. 


\section{Introdução}

A cada ano, cerca de 10 milhões de recém-nascidos, ao redor do mundo, requerem algum tipo de ressuscitação, e mais de um milhão morrem de complicações de asfixia ao nascer ${ }^{1}$. Os balões auto-infláveis são os dispositivos mais utilizados na ventilação pulmonar mecânica manual, sendo recomendados no curso de reanimação neonatal da Sociedade Brasileira de Pediatria2 ${ }^{2}$. Trabalhos têm demonstrado que a utilização do balão pode acarretar variação do volume corrente (VC) e do pico de pressão inspiratória (PIP), conseguidos durante cada insuflação pulmonar ${ }^{3}$. Mondolfi et al. ${ }^{4}$ encontraram grande variação no VC, no PIP e no volume minuto obtidos por profissionais de saúde em manequim de reanimação pediátrico. Baskett et al. ${ }^{5}$ mediram o VC percebido como adequado durante ressuscitação, a partir da expansão torácica, e observaram que é variável. Björklund et al. ${ }^{6}$ demonstraram que a utilização de seis ciclos ventilatórios com grandes volumes no início da ventilação de carneiros prematuros reduz a resposta ao surfactante instilado no período neonatal imediato. Ingimarsson et al. $^{7}$ demonstraram que cinco insuflações pulmonares com VC de $20 \mathrm{~mL} / \mathrm{kg}$ em carneiros prematuros, antes de receberem surfactante exógeno, afetam a distribuição intrapulmonar do surfactante, tornando-a muito desigual, com a distribuição da quase totalidade do medicamento para as áreas dependentes dos pulmões. Wada et al. ${ }^{8}$ observaram que a utilização de maiores VC aplicados aos pulmões de carneiros prematuros, logo após o nascimento, pode causar lesões pulmonares e prejudicar a resposta ao surfactante exógeno.

O objetivo do presente estudo é avaliar o desempenho de médicos em ventilação mecânica manual de carneiros prematuros, com a utilização de balão auto-inflável, por meio do estudo do PIP, do VC e da freqüência respiratória (FR) obtidos durante o procedimento.

\section{Materiais e métodos}

Foi um estudo experimental descritivo, realizado no Laboratório de Fisiologia Respiratória da Universidade.

Cinco carneiros prematuros, de ambos os sexos, provenientes de ovelhas saudáveis, foram admitidos no estudo. A observação de malformações maiores em qualquer animal durante qualquer fase do estudo determinava a sua exclusão.

Dentre 35 neonatologistas considerados experientes por trabalharem em unidade de terapia intensiva (UTI) neonatal pública e/ou privada, 10 foram selecionados utilizando tabela de números aleatórios. Foram considerados experientes aqueles que não eram recém-formados, tinham residência em pediatria e/ou neonatologia e que tinham exercício atual na atividade, com familiaridade em reanimação neonatal. Todos os médicos assinaram termo de consentimento livre e esclarecido.

Os médicos foram escalados em duplas, formadas de maneira aleatória, e cada dupla promoveu ventilação em um carneiro prematuro durante um período de 45 minutos. A utilização de duplas objetivou o revezamento na ventila- ção, para reduzir os riscos de que o cansaço interferisse nos resultados do procedimento. Assim, cada médico produziu de 20 a 25 minutos de ventilação, em períodos alternados de 5 minutos.

As ovelhas e os carneiros prematuros foram abordados de acordo com o descrito por Wada et al. ${ }^{8}$. Resumidamente, ovelha grávida, com $132( \pm 1)$ dias de idade gestacional (termo aos 150 dias de gestação), foi pré-anestesiada com $1 \mathrm{~g}$ de ketamina intramuscular, e feita anestesia epidural usando $10 \mathrm{~mL}$ em uma proporção $1: 1$ (volume a volume) de lidocaína a $2 \%$ e bupivacaína a $0,5 \%$. A ovelha foi intubada utilizando um tubo endotraqueal (TET) com balão, para propiciar melhor controle da ventilação. O carneiro era retirado via cesariana, pesado e colocado sob unidade de calor irradiante (BA Matrix SC, Olidef CZ, Ribeirão Preto, SP, Brasil). Após, era secado e procedia-se à laringoscopia direta; a traquéia era aspirada para retirar excesso de líquido alveolar, e inseria-se um TET com balão, número 4.0 ou 4.5 (que era de tamanho adequado ao carneiro prematuro); então, iniciava-se a ventilação. O balonete do TET era enchido com ar, objetivando eliminar escapes por sua parede externa. Ketamina (10 mg/kg) e acepromazina $(0,1 \mathrm{mg} / \mathrm{kg})$ foram aplicadas por via intramuscular para permitir comando total da ventilação.

Em cada animal recém-nascido, foram cateterizadas veia e artéria umbilicais, com a extremidade dos cateteres posicionados em veia cava inferior e aorta torácica, respectivamente. Os acessos profundos prestaram-se à infusão de glicose a $5 \%$, em taxa equivalente a $100 \mathrm{~mL} / \mathrm{kg} / \mathrm{dia}$, infusão eventual de soro fisiológico para correção de hipotensão arterial e para coleta de gasometrias (arterial) e controle da pressão arterial sistólica e diastólica, além da pressão venosa central. O controle e registro das pressões foram feitos através de um polígrafo (Model 7C, Grass ${ }^{\circledR}$ Instrument Co., Quincy, MA, EUA), a partir de dois transdutores (P23Db Gould Statham ${ }^{\circledR}$, EUA).

Entre a conexão paciente do balão e a cânula traqueal, havia uma saída lateral e um pneumotacógrafo (Fleisch n० 0). O pneumotacógrafo era conectado a um transdutor diferencial (PT5A, Grass, Quincy, MA, EUA), que permitiu o registro e a determinação do fluxo aéreo, o que, por sua vez, permitia o cálculo do volume. A saída lateral era conectada a um transdutor absoluto de pressão (Statham, Gould, EUA), que permitiu o registro e a determinação da pressão traqueal (PIP).

Todos os transdutores estavam ligados ao polígrafo, onde os sinais eram filtrados e amplificados. Do polígrafo, os sinais seguiam para um módulo condicionador de sinais biológicos, desenhado para medida da mecânica ventilatória (Emgsystem do Brasil, São José dos Campos, São Paulo, Brasil), e então para um microcomputador, no qual, através de um conversor analógico-digital (CAD1232, Lynx Tecnologia Eletrônica, São Paulo, SP), os sinais eram digitalizados para armazenamento e posterior análise. O software utilizado para armazenamento e análise dos sinais coletados foi o AqDados 5 versão para Windows ${ }^{\circledR}$. A freqüência de amostragem utilizada em todo o experimento foi de $200 \mathrm{~Hz}$. 
O balão auto-inflável utilizado era novo, de tamanho neonatal, de $280 \mathrm{~mL}$, com bolsa reservatória de baixa complacência para permitir $\mathrm{FiO}_{2}$ de $100 \%$, marca Lifesa$\operatorname{ver}^{\circledR}$ (Hudson $\mathrm{RCI}^{\circledR}$, Temecula, $\mathrm{CA}, \mathrm{EUA}$ ). A fonte de oxigênio tinha pressão de saída de $3,5 \mathrm{kgf} / \mathrm{cm}^{2}$, e o fluxo aferente de oxigênio era de $10 \mathrm{~L} / \mathrm{min}$, mantendo a bolsa reservatória de baixa complacência sempre com gases. Para efeito de estudo, o balão teve sua válvula de alívio bloqueada.

Os médicos foram instruídos a realizar a ventilação pulmonar segundo seus critérios habituais para otimização do processo, dispondo da observação clínica geral e da visualização da expansibilidade da caixa torácica dos animais. Não tinham acesso visual ou qualquer outra informação sobre os parâmetros de mecânica ou de troca gasosa estudados.

Os dados foram coletados e armazenados de maneira contínua, registrando os traçados de pressão e fluxo aéreo em função do tempo. Para análise dos dados, foram selecionados os exatos últimos 50 segundos de cada 5 minutos. A FR por minuto era obtida multiplicando por 1,2 o número de ciclos analisados em 50 segundos. O VC/kg era obtido a partir do cálculo do VC em cada curva de fluxo dividido pelo peso do animal.

Os dados foram avaliados utilizando-se o Excel ${ }^{\circledR}$ (Microsoft $^{\circledR}$ ) e SPSS ${ }^{\circledR}$.

O protocolo foi submetido à apreciação do comitê de ética do uso animal da Universidade, que o aprovou.

\section{Resultados}

A mediana do PIP foi de $39,8 \mathrm{cmH}_{2} \mathrm{O}\left(\mathrm{IQ}_{25-75 \%} 30,2-\right.$ $47,2)$. PIP menor que $20 \mathrm{cmH}_{2} \mathrm{O}$ ocorreu em $1,1 \%$ das vezes e maior que 40 em 49,1\%. Sete em 10 médicos propiciaram mais de seis PIP maiores que $40 \mathrm{cmH}_{2} \mathrm{O}$. A mediana do
$\mathrm{VC} / \mathrm{kg}$ foi de $17,8 \mathrm{~mL}\left(\mathrm{IQ}_{25-75 \%} 14,1-22,4\right)$, sendo menor que $5 \mathrm{~mL}$ em $0,1 \%$ das vezes, igual ou maior que $10 \mathrm{~mL}$ em $90,2 \%$ e igual ou maior que $20 \mathrm{~mL}$ em $37,7 \%$. Todos os médicos impuseram cinco ou mais ciclos ventilatórios com $\mathrm{VC} / \mathrm{kg}$ de $20 \mathrm{~mL}$ ou mais. A FR situou-se entre 30 e 60 ciclos/ minuto em $65,9 \%$ das vezes, sendo menor que 30 em $6,8 \%$ e maior que 60 em $27,3 \%$ das vezes. A Tabela 1 apresenta as medianas e os intervalos interquartis de cada médico, bem como os resultados globais.

\section{Discussão}

Neste experimento, foram analisadas 1.872 curvas para o PIP e VC, as quais demonstraram que houve grande variabilidade nos parâmetros avaliados. Tanto a pressão quanto o $\mathrm{VC} / \mathrm{kg}$ alcançaram níveis elevados, quando comparados aos sugeridos em guias de ventilação manual. Em $49,1 \%$ das vezes, os médicos ventilaram os carneiros com PIP maior que $40 \mathrm{cmH}_{2} \mathrm{O}$; o ILCOR $^{9}$ e o curso de reanimação neonatal ${ }^{2}$ sugerem que o PIP máximo seja de $40 \mathrm{cmH}_{2} \mathrm{O}$. A demonstração da variabilidade das pressões de ventilação ao utilizar o balão não é novidade; Mondolfi et al. ${ }^{4}$ demonstraram PIP médio de $35 \pm 19 \mathrm{~cm} \mathrm{H}_{2} \mathrm{O}$, utilizando manequim de reanimação pediátrico. Nosso estudo mostra, na tabela, que todos os médicos impuseram variações nos valores, tanto de pressão quanto de $\mathrm{VC} / \mathrm{kg}$.

As limitações dos balões auto-infláveis como instrumento de ventilação já estão bem documentadas ${ }^{10-15}$. Por exemplo, no estudo de Barnes et al. ${ }^{14}$, avaliaram-se o desempenho e a segurança de 10 ressuscitadores manuais descartáveis, sendo quatro deles pediátricos, e concluiu-se que apenas um dos aparelhos pediátricos analisados e três dos adultos respondiam a todos os requisitos exigidos pela norma F920-93 da American Society for Testing and Materials (ASTM) ${ }^{16}$.

Tabela 1 - Valores de pico de pressão, freqüência respiratória e volume corrente de cada participante e do grupo todo

\begin{tabular}{|c|c|c|c|c|c|}
\hline Médico & $\begin{array}{l}\text { PIP desejável* } \\
\qquad \mathrm{cmH}_{2} \mathrm{O}\end{array}$ & $\begin{array}{c}\text { PIP observado } \\
\left(\mathrm{cmH}_{2} \mathrm{O}\right) \\
\text { Mediana }\left(\mathrm{IQ}_{25-75 \%}\right)\end{array}$ & $\begin{array}{c}\text { FR } \\
\text { desejável* }\end{array}$ & $\begin{array}{c}\text { FR observada } \\
\text { (mpm) } \\
\text { Mediana }\left(\mathrm{IQ}_{\mathbf{2 5 - 7 5} \%}\right)\end{array}$ & $\begin{array}{c}\text { VC observado } \\
(\mathrm{mL} / \mathbf{k g}) \\
\text { Mediana }\left(\mathrm{IQ} \mathbf{2}_{25-75 \%}\right)\end{array}$ \\
\hline 1 & $20-40$ & $43,1(40,1-49)$ & $30-60$ & $56(56-61)$ & $16(14,1-18,9)$ \\
\hline 2 & $20-40$ & $44,6(37,6-51,5)$ & $30-60$ & $84(79-89,7)$ & $16(12,5-22,1)$ \\
\hline 3 & $20-40$ & $27,1(25,2-29,8)$ & $30-60$ & $46(46-48)$ & $11,6(9,6-16,4)$ \\
\hline 4 & $20-40$ & $24,1(22-26,3)$ & $30-60$ & $43(41,48,5)$ & $9,1(7,8-14,2)$ \\
\hline 5 & $20-40$ & $35,8(29,1-38,6)$ & $30-60$ & $38(36-39)$ & $16,9(14,9-19,3)$ \\
\hline 6 & $20-40$ & $30,1(26,2-32,9)$ & $30-60$ & $36(33,8-39)$ & $18,5(16,3-21,9)$ \\
\hline 7 & $20-40$ & $47,3(43,8-50,2)$ & $30-60$ & $62(57-62)$ & $24,3(22,7-26)$ \\
\hline 8 & $20-40$ & $47,7(43,8-54,2)$ & $30-60$ & $63(57,7-65,2)$ & $22,9(20,9-24,4)$ \\
\hline 9 & $20-40$ & $33,5(30,2-38,5)$ & $30-60$ & $32(31,5-34,7)$ & $16,5(15,2-18)$ \\
\hline 10 & $20-40$ & $43,7(34,5-47,9)$ & $30-60$ & $26(25,5-27,2)$ & $17,2(15,8-19,6)$ \\
\hline Todos & $20-40$ & $39,8(30,2-47,2)$ & $30-60$ & $46(37,5-61,2)$ & $17,8(14,1-22,4)$ \\
\hline
\end{tabular}

$\mathrm{PIP}=$ pico de pressão inspiratória; $\mathrm{FR}=$ freqüência respiratória, em ciclos $/$ minuto; $\mathrm{VC}=$ volume corrente $(\mathrm{mL} / \mathrm{kg})$. * ILCOR ${ }^{9}$

Os valores foram expressos através da mediana e do respectivo intervalo interquartil $25-75 \%$. 
No presente experimento, optou-se por realizar ventilação manual mantendo o balão com a válvula de alívio bloqueada. Esse tipo de válvula é regulado pelo fabricante, em concordância com a norma F920-93 ${ }^{16}$, para que libere pressão quando atingir $40 \pm 5 \mathrm{cmH}_{2} \mathrm{O}$, no caso do modelo neonatal. No entanto, existem falhas no desempenho dessas válvulas 10,15 , que, mesmo testadas, podem aliviar pressão em níveis diferentes daqueles recomendados. 0 trabalho de Finer et al. ${ }^{15}$, por exemplo, estudando três marcas diferentes de balão neonatal, evidenciou que a pressão de abertura variou de 41 a $72 \mathrm{cmH}_{2} \mathrm{O}$ para um deles, de 51 a $97 \mathrm{cmH}_{2} \mathrm{O}$ para o segundo e, ainda, de 38 a 106 $\mathrm{cmH}_{2} \mathrm{O}$ para o terceiro balão, valores muito diferentes daqueles que os próprios fabricantes informavam ter calibrado os referidos equipamentos $\left(35 \mathrm{cmH}_{2} \mathrm{O}, 43-60 \mathrm{cmH}_{2} \mathrm{O}\right.$ e $40 \mathrm{cmH}_{2} \mathrm{O}$, respectivamente).

Mesmo utilizando um balão com a válvula de alívio bloqueada, os médicos não conseguiram, em $49 \%$ das vezes, se manter dentro do limite máximo de pressão sugerida pelo ILCOR ${ }^{9}$, e $70 \%$ deles ventilaram mais que seis vezes com pressão acima de $40 \mathrm{cmH}_{2} \mathrm{O}$, considerando somente as curvas analisadas ( 50 segundos de cada 5 minutos). É possível especular que a presença da válvula de alívio funcionando faria com que os médicos ultrapassassem mais vezes o PIP de $40 \mathrm{cmH}_{2} \mathrm{O}$, se ela estivesse regulada em $45 \mathrm{cmH}_{2} \mathrm{O}$ (ainda dentro do preconizado pela ASTM), porque teriam a válvula de alívio como fator de segurança, retirando de si mesmos a responsabilidade ou a preocupação com essa limitação.

A mediana para o $\mathrm{VC} / \mathrm{kg}$ foi de $17,8 \mathrm{~mL}\left(\mathrm{IQ}_{25-75 \%} 14,1\right.$ $22,4)$, sendo igual ou maior que $10 \mathrm{~mL}$ em $90,2 \%$ e igual ou maior que $20 \mathrm{~mL}$ em 37,7\%. Aqui também Mondolfi et al. 4 identificaram grande variabilidade: média de $25 \pm 10 \mathrm{~mL}$ (limites de 3 a $103 \mathrm{~mL}$ ). As razões para essa variabilidade são dependentes não somente das pressões de ventilação como também da complacência do sistema respiratório.

Os danos potenciais de números como os que foram encontrados no presente estudo estão demonstrados na literatura. Wada et al. 8 verificaram que, em carneiros prematuros, VC de $10 \mathrm{~mL} / \mathrm{kg}$ durante os primeiros 30 minutos após o nascimento resultava em prejuízos na mecânica ventilatória e nas trocas gasosas nas primeiras 6 horas de ventilação pulmonar, quando comparados a carneiros ventilados em igual período, mas que haviam sido ventilados nos primeiros 30 minutos com VC de $5 \mathrm{~mL} / \mathrm{kg}$, ainda que recebessem surfactante. No presente experimento, em 90,2\% das vezes, os médicos impuseram aos carneiros um $\mathrm{VC} / \mathrm{kg}$ igual ou maior que $10 \mathrm{~mL}$. Os estudos de Ingimarsson et al. ${ }^{7}$ demonstraram que cinco insuflações pulmonares com altos volumes corrente $(20 \mathrm{~mL} / \mathrm{kg})$ em carneiros prematuros, antes de receberem surfactante exógeno, afetaram a sua distribuição intrapulmonar. No presente estudo, todos os médicos impuseram, pelo menos, cinco ciclos ventilatórios com VC/kg de $20 \mathrm{~mL}$ ou mais.

A vigilância da adequação de VC é habitualmente feita através da expansão torácica. Porém, em acordo com o que demonstrou Baskett et al. ${ }^{5}$, a percepção da expansão torácica não parece suficiente para adequar o VC ideal, pelo menos em adultos. As pressões utilizadas por Hird et al. ${ }^{17}$ até que os recém-nascidos prematuros tivessem uma "expansão torácica adequada" variou de 14 a $30 \mathrm{cmH}_{2} \mathrm{O}$, e não se correlacionaram com o peso dos prematuros, nem com a idade gestacional. No presente experimento, os valores de PIP e VC/kg encontrados demonstram que os médicos tiveram pontos de vista diferentes sobre o que é expansão torácica adequada.

É consenso que a "expansão física dos pulmões, com estabelecimento da capacidade residual funcional e aumento na pressão parcial de oxigênio mediam o crítico decréscimo na resistência vascular pulmonar e resultam no aumento do fluxo sangüíneo pulmonar após o nascimento (...) falha na expansão adequada dos espaços alveolares pode resultar em shunt intrapulmonar com resultante hipoxemia"9. Dados da literatura6-8 indicam que há um limite, talvez estreito, para definir essa adequação, pois precisa ser bem dimensionada para não ultrapassar a medida exata em que o pulmão servirá como mediador da troca gasosa, sem prejuízo da sua função no futuro próximo. O desejável é uma permanente troca gasosa adequada. A aplicação de pressões elevadas em tempo prolongado foi sugerida 18 como a garantia de que a capacidade residual funcional mais elevada seria rapidamente adquirida, melhorando a oxigenação, sendo rapidamente incorporada na prática clínica como o desejado. Atualmente, é uma prática não universalmente recomendada ${ }^{9}$, e tanto os trabalhos de Björklund et al. ${ }^{6}$ e Ingimarsson et al. ${ }^{7}$ quanto o de Wada et al. ${ }^{8}$ alertam para o seu perigo. Percebe-se na literatura a preocupação com o fato de que a displasia broncopulmonar seria uma doença que começa na sala de parto, tendo como causa uma ventilação extremamente agressiva, causando hipocapnia e volutrauma1,19,20.

A FR foi a variável do processo de ventilação em que os médicos apresentaram melhor desempenho, situando-se na faixa desejada em $65,9 \%$ das vezes.

Este experimento contém limitações. As condições de estresse dos médicos não foram semelhantes àquelas encontradas na realidade do atendimento do recém-nascido humano, e os números encontrados podem estar até melhores do que os que seriam encontrados em um atendimento real a um recém-nascido. O número de animais utilizados no estudo, assim como o número de médicos, pode ser considerado pequeno. Para o fato deste estudo ter utilizado animais, pondera-se duas coisas: o animal mais utilizado como modelo para o recém-nascido humano tem sido o carneiro $6-8,21,22$, e a pesquisa com esse desenho não pode ser feita em humanos por limitações éticas. O número de animais por grupo tem variado de quatro a oito em diversos experimentos de reconhecida qualidade ${ }^{6-8,21,22}$. No presente experimento, o número de médicos permitiu que analisássemos 1.872 eventos de cada (PIP e VC). Os 10 médicos avaliados fizeram o curso de reanimação neonatal e foram selecionados aleatoriamente, entre 35 médicos intensivistas neonatais identificados como experientes, na tentativa de restringir vícios de seleção; porém, sempre existe a possibilidade de viés, porque os 35 neonatologistas não representam todos os médicos classificáveis como "neonatologistas experientes". Consideramos a tecnologia 
empregada no experimento com nível de sofisticação suficiente para a fidelidade dos dados; ainda assim, os dados aqui demonstrados e as conclusões apontadas devem ser avaliados com as reservas apropriadas aos estudos experimentais em animais.

Os resultados do presente estudo indicam que os médicos, mesmo experientes e treinados, podem não obter sucesso na ventilação manual de carneiros prematuros utilizando balão auto-inflável, considerando as normas internacionais estabelecidas. Ocorreu grande variabilidade nos valores do PIP e do VC/kg, que muitas vezes foram elevados e alcançaram níveis habitualmente indutores de biotrauma. Para a FR, os valores conseguidos foram adequados na maioria das vezes.

\section{Agradecimentos}

Os autores agradecem à Hercilia Maria Nogueira de Resende e Patrícia Carvalho Baião Câmara, pela revisão ortográfica dos textos.

\section{Conflito de interesse}

Jefferson Guimarães de Resende é inventor e proprietário de patente de invenção de um equipamento de ventilação pulmonar mecânica manual.

\section{Referências}

1. Wiswell TE. Neonatal resuscitation. Respir Care. 2003;48:288-94.

2. Sociedade Brasileira de Pediatria. Manual do curso de reanimação neonatal. São Paulo: Universidade Federal de São Paulo Escola Paulista de Medicina; 1996. [Traduzido do inglês. Copyright by American Heart Association.]

3. Barnes TA. Emergency ventilation techniques and related equipment. Respir Care. 1992;37:673-90; discussion 690-4.

4. Mondolfi AA, Grenier BM, Thompson JE, Bachur RG. Comparison of self-inflating bags with anesthesia bags for bag-mask ventilation in the pediatric emergency department. Pediatr Emerg Care. 1997;13:312-6.

5. Baskett P, Nolan J, Parr M. Tidal volumes which are perceived to be adequate for resuscitation. Resuscitation. 1996;31:231-4.

6. Björklund LJ, Ingimarsson J, Curstedt $T$, John J, Robertson B, Werner $\mathrm{O}$, et al. Manual ventilation with a few large breaths at birth compromises the therapeutic effect of subsequent surfactant replacement in immature lambs. Pediatr Res. 1997;42:348-55.

7. Ingimarsson J, Björklund LJ, Curstedt $T$, Jonson $B$, Larsson $A$, Robertson B, et al. Uneven distribution of exogenous surfactant after hyperinflation of the lungs at birth in immature lambs. 2001 Pediatric Academic Societies Annual Meeting; 2001 Apr 28-May 1; Baltimore, Maryland: US. Abstract 2200.
8. Wada K, Jobe AH, Ikegami M. Tidal Volume effects on surfactant treatment responses with the initiation of ventilation in preterm lambs. J Appl Physiol. 1997;83:1054-61.

9. Kattwinkel J, Niermeyer S, Nadkarni V, Tibballs J, Phillips B, Zideman $D$, et al. An advisory statement from the Pediatric Working Group of the International Liaison Committee on Resuscitation Pediatrics. 1999; 103: e56. http://www.pediatrics. org/cgi/content/full/103/4/e56.

10. Connors R, Kisson N, Tiffin N, Frewer TC. An evaluation of the physical and functional characteristics of infant resuscitators. Pediatr Emerg Care. 1993;9:104-7.

11. Mills PJ, Baptiste J, Preston J, Barnas GM. Manual resuscitators and spontaneous ventilation - an evaluation. Crit Care Med. 1991;19:1425-31.

12. Martell RJ, Soder CM. Laerdal infant resuscitators are unreliable as free-flow oxygen delivery devices. Am J Perinatol 1997;14: 347-51.

13. Hess $D$, Hirsch $C$, Marquis-D'Amico $C$, Kacmarek RM. Imposed work and oxygen delivery during spontaneous breathing with adult disposable manual ventilators. Anesthesiology. 1994;81:1256-63.

14. Barnes TA, McGarry WP 3rd. Evaluation of ten disposable manual resuscitators. Respir Care. 1990;35:960-8.

15. Finer NN, Barrington KJ, Al-Fadley F, Peters KL. Limitations of self-inflating resuscitators. Pediatrics 1986;77:417-20.

16. American Society for Testing and Materials. Standard specification for minimum performance and safety requirements for resuscitators intended for use with humans. ASTM International. 1993; F 920-93.

17. Hird MF, Greenough A, Gamsu HR. Inflating pressures for effective resuscitation of preterm infants. Early Hum Dev. $1991 ; 26: 69-72$.

18. Vyas $\mathrm{H}$, Milner AD, Hopkin IE, Boon AW. Physiologic responses to prolonged and slow-rise inflation in the resuscitation of the asphyxiated newborn infant. J Pediatr. 1981;99:635-9.

19. Sharek PJ, Baker R, Litman F, Kaempf J, Burch K, Schwarz E, et al. Evaluation and development of potentially better practices to prevent chronic lung disease and reduce lung injury in neonates. Pediatrics. 2003;111:e426-31. http://pediatrics.aappublications. org/cgi/reprint/111/4/SE1/e426.

20. Burch K, Rhine W, Baker R, Litman F, Kaempf JW, Schwarz E, et al. Implementing potentially better practices to reduce lung injury in neonates. Pediatrics. 2003;111:e432-6. http:// pediatrics.aappublications.org/cgi/reprint/111/4/SE1/e432.

21. Naik AS, Kallapur SG, Bachurski, Jobe AH, Michna J, Kramer BW, et al. Effects of ventilation with different positive end-expiratory pressures on cytokine expression in the preterm lamb lung. Am J Respir Crit Care Med. 2001;164:494-8.

22. Michna J, Jobe AH, Ikegami M. Positive end-expiratory pressure preserves surfactant function in preterm lambs. Am J Respir Crit Care Med. 1999;160:634-9.

Correspondência:

Jefferson Guimarães de Resende

SHIN QL 10, conjunto 1, casa 14, Lago Norte

CEP 71525-015 - Brasília, DF

Tel.: (61) 3368.4665

Fax: (61) 3386.7481

E-mail: jeffersoncfr@hotmail.com 